\title{
RADIALLY SYMMETRIC SOLUTIONS TO A SUPERLINEAR DIRICHLET PROBLEM IN A BALL WITH JUMPING NONLINEARITIES
}

\author{
ALFONSO CASTRO AND ALEXANDRA KUREPA
}

\begin{abstract}
Let $p, \varphi:[0, T] \rightarrow R$ be bounded functions with $\varphi>0$. Let $g: \mathbf{R} \rightarrow \mathbf{R}$ be a locally Lipschitzian function satisfying the superlinear jumping condition: (i) $\lim _{u \rightarrow-\infty}(g(u) / u) \in \mathbf{R}$, (ii) $\lim _{u \rightarrow \infty}\left(g(u) / u^{1+\rho}\right)=\infty$ for some $\rho>0$, and (iii) $\lim _{u \rightarrow \infty}(u / g(u))^{N / 2}(N G(\kappa u)-((N-2) / 2) u \cdot g(u))=\infty$ for some $\kappa \in(0,1]$ where $G$ is the primitive of $g$. Here we prove that the number of solutions of the boundary value problem $\Delta u+g(u)=p(\|x\|)+c \varphi(\|x\|)$ for $x \in \mathbf{R}^{N}$ with $\|x\|<T, u(x)=0$ for $\|x\|=T$, tends to $+\infty$ when $c$ tends to $+\infty$. The proofs are based on the "energy" and "phase plane" analysis.
\end{abstract}

\section{INTRODUCTION}

In this paper we consider the existence of solutions to the Dirichlet problem

$$
\begin{gathered}
\Delta u+g(u)=p(\|x\|)+c \varphi(\|x\|), \quad x \in \Omega, \\
u=0, \quad x \in \delta \Omega,
\end{gathered}
$$

where $\Omega$ is the ball of radius $T$ in $\mathbf{R}^{N}$ centered at the origin, $g: \mathbf{R} \rightarrow \mathbf{R}$ is a locally Lipschitzian function, $p \in L^{2}(\Omega), c \in \mathbf{R}$, and $\varphi:[0, T] \rightarrow \mathbf{R}$ is a differentiable function with

$$
\varphi>0 \text { on }[0, T] \text {. }
$$

In addition we assume that the problem is superlinear with jumping nonlinearities, i.e., that there exist real numbers $M$ and $\rho>0$ such that

$$
\begin{gathered}
\lim _{u \rightarrow-\infty} \frac{g(u)}{u}=M, \\
\lim _{u \rightarrow \infty} \frac{g(u)}{u^{1+\rho}}=\infty .
\end{gathered}
$$

For the sake of simplicity of the proofs we assume, without loss of generality, that

$$
\begin{aligned}
& g(0)=0 \text { and } g \text { is strictly increasing, } M>0, \\
& p \in L^{\infty}(\Omega), \text { and } 1 \leq \varphi(t) \leq 2 \text { for all } t \in[0, T] .
\end{aligned}
$$

Received by the editors June 8, 1987 and, in revised form, March 29, 1988.

1980 Mathematics Subject Classification (1985 Revision). Primary 35J65; 34A10.

Key words and phrases. Dirichlet problem, superlinear jumping nonlinearity, singular differential equation, radially symmetric solutions. 
In order to state our main results we introduce the following notations:

$$
\begin{gathered}
L(\kappa, u)=N G(\kappa u)-((N-2) / 2) u g(u), \\
L(\kappa)=\lim _{u \rightarrow \infty} L(\kappa, u)(u / g(u))^{N / 2},
\end{gathered}
$$

where $G(u)=\int_{0}^{u} g(v) d v$ and $\kappa \in(0,1]$.

Our main result is

Theorem A. If (1.2)-(1.5) hold and $L(\kappa)=\infty$ for some $\kappa \in(0,1]$, then there exists a positive integer $J$ and an increasing sequence $\left\{c_{j}: j=J, J+1, \ldots\right\}$ tending to $\infty$ such that for $c>c_{j}$ the equation (1.1) has a radially symmetric solution $u_{j}$ with $u_{j}(0)<0$, and $u_{j}$ has $j$ interior nodal surfaces. In particular if $c>c_{j}$ then the equation (1.1) has $j-J$ radially symmetric solutions with $u(0)<0$.

Theorem $\mathrm{A}$ is in the spirit of studying boundary value problems for which the interval $\left(\lim _{u \rightarrow-\infty}(g(u) / u), \lim _{u \rightarrow \infty}(g(u) / u)\right)$ contains at least one eigenvalue of $-\Delta$ with Dirichlet boundary conditions. Such a problem is called superlinear (resp. sublinear) if $\lim _{u \rightarrow \infty}(g(u) / u)=\infty$ (resp. $\left.\lim _{u \rightarrow \infty}(g(u) / u)<\infty\right)$. The one-dimensional superlinear version of Theorem $A$ is given in [5], and it motivated our result (see also [4 and 13]). For studies on the sublinear case we refer the reader to [11] and references therein.

The proof of Theorem A is based on the shooting method that we have also used in [3]. We study the singular initial value problem

$$
\begin{gathered}
u^{\prime \prime}+\frac{N-1}{t} u^{\prime}+g(u)=p(t)+c \varphi(t), \quad t \in[0, T], \\
u(0)=d, \quad u^{\prime}(0)=0,
\end{gathered}
$$

where $d \in \mathbf{R}$. A simple argument based on the contraction mapping principle shows that (1.8) has a unique solution $u(t, d, c)$ on the interval $[0, T]$ depending continuously on $(d, c)$ (see [3, Lemma 2.1$]$ ). Radially symmetric solutions of (1.1) are the solutions of (1.8) satisfying

$$
u(T, d, c)=0 \text {. }
$$

We analyze the energy of the corresponding solutions, i.e., we analyze the function

$$
E(t, d, c)=\frac{\left(u^{\prime}(t, d, c)\right)^{2}}{2}+G(u(t, d, c)) .
$$

In order to count the number of zeros of the solutions to (1.8) we show that

$$
E\left(t,-c^{\zeta}, c\right)>0
$$

for $\zeta>(N+1) /(N+4)$ and $c$ sufficiently large. In turn (1.11) implies that in the $\left(u, u^{\prime}\right)$ plane, for $c$ sufficiently large and for $d \leq-c^{(N+1) /(N+4)}$, a continuous argument function $\psi(t, d, c)$ can be defined. The function $\psi(t, d, c)$ is such 
that $\psi(t, d, c)=i \pi+\pi / 2$ ( $i$ an integer) if and only if $u(t, d, c)=0$. We show that (see Lemma 4.1 and Lemma 2.7)

$$
\limsup _{-d \rightarrow \infty} \psi(T, d, c)=S,
$$

and that

$$
\liminf _{c \rightarrow \infty} \psi\left(T,-c^{\xi}, c\right)=\infty,
$$

where $S \in \mathbf{R}$ and $\xi \in[(N+1) /(N+4), 1)$. The intermediate value theorem and (1.13) imply that given any integer $j$ there exists $c_{j}$ such that if $c>c_{j}$ then

$$
\psi\left(T,-c^{\xi}, c\right) \geq j \pi+\pi / 2 .
$$

By combining (1.12), (1.14) and the intermediate value theorem we see that if $c$ is sufficiently large and $j \geq J:=[(S-\pi / 2) / \pi]+1$, then there exist numbers $d_{J}<d_{J+1}<\cdots<d_{j}$ with

$$
\psi\left(t, d_{i}, c\right)=i \pi+\pi / 2, \quad i \in\{J, J+1, \ldots, j\} .
$$

Hence

$$
u_{i}\left(T, d_{i}, c\right)=0, \quad i \in\{J, J+1, \ldots, j\} .
$$

Thus if $c$ is sufficiently large, then $(1.1)$ has $j-J$ radially symmetric solutions.

Remarks. (i) If $\Omega$ is a ring of the form $\left\{x \in \mathbf{R}^{N}: a \leq\|x\| \leq b\right\}$, then the equation in (1.8) is no longer singular and thus the problem reduces to the one studied in [5].

(ii) Condition (1.2) can be considerably weakened. For example, it is easy to verify that if $\varphi>0$ on $[0, \varepsilon)$ and $\varphi=0$ on $[\varepsilon, T]$, then Theorem A holds.

\section{Phase-Plane analysis}

Let $\left(u(t, d, c), u^{\prime}(t, d, c)\right) \neq(0,0)$ for all $t \in[0, \underline{t})$. By defining $r^{2}(t, d, c)$ $=u^{2}(t, d, c)+\left(u^{\prime}(t, d, c)\right)^{2}$ we see that for $d<0$ there exists a unique continuous argument function $\psi(t, d, c), t \in[0, \underline{t})$, such that

$$
\begin{gathered}
u(t, d, c)=-r(t, d, c) \cos \psi(t, d, c), \\
u^{\prime}(t, d, c)=r(t, d, c) \sin \psi(t, d, c), \\
\psi(0, d, c)=0 .
\end{gathered}
$$

An elementary calculation shows that

$$
\begin{aligned}
\psi^{\prime}(t, d, c)=\sin ^{2} \psi(t, d, c) & \\
& -\frac{\left(g(u(t, d, c))+\frac{N-1}{t} u^{\prime}(t, d, c)-c \varphi(t)-p(t)\right) \cos \psi(t, d, c)}{r(t, d, c)} .
\end{aligned}
$$

From this formula follows: 
Remark 2.1. If $r(t, d, c)>0$ for all $t \in[0, T]$, and $\psi(\hat{t}, d, c)=(2 k+1) \pi / 2$ for some $\hat{t} \in[0, T]$ and some integer $k$, then $\psi(t, d, c)>(2 k+1) \pi / 2$ for all $t>\hat{t}$. In particular, $\psi(t, d, c)>-\pi / 2$ for all $t \in[0, T]$.

Now let $\gamma$ be such that

$$
\gamma_{1}:=1-12 \varepsilon \leq \gamma \leq 1-10 \varepsilon:=\gamma_{2},
$$

where $\varepsilon$ is defined by

$$
\varepsilon=\frac{\rho}{4(6 N+6 N \rho+8+8 \rho)} .
$$

Elementary calculations show that

$$
\text { (a) } 2 \gamma>2 \gamma+(\gamma-1) N>2 \gamma+\frac{3}{2}(\gamma-1) N>1+\frac{1}{1+\rho} \text {, }
$$
(b) $\gamma+\varepsilon<1$,
(c) $\gamma>\varepsilon+\frac{1}{1+\rho}$
(d) $3 \gamma-2-\frac{\varepsilon}{2}>\frac{2 \gamma}{2+\rho}$,
(e) $2 \gamma-1>\frac{N+1}{N+4}$.

Let $u(t, d, c):=u(t)$ be a solution to (1.8). Since the following constant appears often in this section we let

$$
Q:=Q(N):=\frac{[\sqrt{2 N}+512 N]^{2}}{2 N} .
$$

Throughout the paper $k$ will denote a nonnegative integer.

Lemma 2.2. There exists $C_{1}$ such that if $c>C_{1}, E\left(t_{1}\right)=c^{2 \gamma}, u\left(t_{1}\right)>-\alpha c^{2 \gamma-1}$ and $\psi\left(t_{1}\right) \in[2 k \pi, 2 k \pi+\pi / 2]$, for some $t_{1} \in(0, T]$ and $\alpha \in[1,64 N]$, then there exists $t_{2} \in\left[t_{1}, t_{1}+2 \alpha \sqrt{N} c^{\gamma-1}\right]$, such that

$$
\psi\left(t_{2}\right)=2 k \pi+\pi / 2,
$$

and

$$
\frac{1}{128} c^{2 \gamma} \leq E\left(t_{2}\right) \leq \frac{(\sqrt{2 N}+8 \alpha)^{2}}{2 N} c^{2 \gamma} \leq Q c^{2 \gamma} .
$$

In particular $E\left(t_{2}\right)=c^{2 \gamma^{\prime}}$ with $\gamma^{\prime} \in[\gamma-(\ln 128) /(2 \ln c), \gamma+(\ln Q) /(2 \ln c)]$.

Proof. Let $t>t_{1}$ be such that $u(s) \leq 0$ for all $s \in\left(t_{1}, t\right]$. Therefore, we have

$$
\begin{aligned}
u^{\prime}(t) & =t^{-N+1}\left[t_{1}^{N-1} u^{\prime}\left(t_{1}\right)+\int_{t_{1}}^{t} s^{N-1}(c \varphi(s)+p(s)-g(u(s))) d s\right] \\
& \geq t^{-N+1} \int_{t_{1}}^{t} s^{N-1}\left(c-\|p\|_{\infty}\right) d s \\
& \geq \frac{c-\|p\|_{\infty}}{N}\left[t-\frac{\left(t_{1}\right)^{N}}{t^{N-1}}\right] \geq \frac{c-\|p\|_{\infty}}{N}\left(t-t_{1}\right) .
\end{aligned}
$$

Thus, for $c$ sufficiently large we infer

$$
\begin{aligned}
u(t) & \geq-\alpha c^{2 \gamma-1}+\frac{c-\|p\|_{\infty}}{2 N}\left(t-t_{1}\right)^{2} \\
& \geq-\alpha c^{2 \gamma-1}+\frac{c}{4 N}\left(t-t_{1}\right)^{2} .
\end{aligned}
$$


This shows that for some $t_{2} \in\left[t_{1}, t_{1}+2 \alpha \sqrt{N} c^{\gamma-1}\right]$ we have

$$
u\left(t_{2}\right)=0,
$$

and $u^{\prime}(s) \geq 0$ for all $s \in\left(t_{1}, t_{2}\right)$. In particular

$$
\psi\left(t_{2}\right)=2 k \pi+\pi / 2 .
$$

By the continuity of $u^{\prime}$ there exists $\tau<t_{1}$ such that $u^{\prime}(\tau)=0$. Since $u\left(t_{1}\right) \geq-\alpha c^{2 \gamma-1}$, then from (1.3) for $u$ sufficiently large, we have that $|g(u)| \leq$ $(M+1)|u|$. Therefore, for $c$ sufficiently large

$$
G\left(u\left(t_{1}\right)\right) \leq[(M+2) / 2] \alpha^{2} c^{4 y-2} .
$$

Thus for $c$ sufficiently large we have

$$
u^{\prime}\left(t_{1}\right) \geq c^{\gamma} .
$$

Hence,

$$
c^{\gamma} \leq u^{\prime}\left(t_{1}\right)=t_{1}^{-N+1} \int_{\tau}^{t_{1}} s^{N-1}(c \varphi(s)+p(s)-g(u(s))) d s \leq \frac{4}{N} c\left(t_{1}-\tau\right),
$$

which implies that

$$
t_{1}-\tau \geq N c^{\gamma-1} / 4
$$

Therefore,

$$
\begin{aligned}
u^{\prime}\left(t_{2}\right) & =t_{2}^{-N+1} \int_{\tau}^{t_{2}} s^{N-1}(c \varphi(s)+p(s)-g(u(s))) d s \\
& \geq \frac{c-\|p\|_{\infty}}{N}\left(t_{2}-\tau\right) \geq \frac{c}{8} c^{\gamma-1} .
\end{aligned}
$$

Thus

$$
E\left(t_{2}\right) \geq \frac{1}{128} c^{2 \gamma}
$$

Now, for $t \in\left(t_{1}, t_{2}\right]$ we have

$$
\begin{aligned}
u^{\prime}(t) & =t^{-N+1}\left[t_{1}^{N-1} u^{\prime}\left(t_{1}\right)+\int_{t_{1}}^{t} s^{N-1}(c \varphi(s)+p(s)-g(u(s))) d s\right] \\
& \leq \sqrt{2} c^{\gamma}+t^{-N+1} \int_{t_{1}}^{t} s^{N-1}\left(2 c+\|p\|_{\infty}+(M+1)\left|u\left(t_{1}\right)\right|\right) d s \\
& \leq \sqrt{2} c^{\gamma}+t^{-N+1} \int_{t_{1}}^{t} s^{N-1}\left(2 c+\|p\|_{\infty}+(M+1) \alpha c^{2 \gamma-1}\right) d s \\
& \leq \sqrt{2} c^{\gamma}+\left(2 c+\|p\|_{\infty}+(M+1) \alpha c^{2 \gamma-1}\right) 2 \alpha \frac{\sqrt{N}}{N} c^{\gamma-1} \\
& \leq \sqrt{2} c^{\gamma}+4 c \frac{2 \alpha}{\sqrt{N}} c^{\gamma-1} \leq \frac{\sqrt{2 N}+8 \alpha}{\sqrt{N}} c^{\gamma},
\end{aligned}
$$


where we have used (1.3), the hypothesis of the lemma and the fact that $t_{2} \in$ $\left[t_{1}, t_{1}+2 \alpha \sqrt{N} c^{\gamma-1}\right]$. Since $E\left(t_{2}\right)=\left(u^{\prime}\left(t_{2}\right)\right)^{2} / 2$ we see that for $c>C_{1}$ we have

$$
E\left(t_{2}\right) \leq \frac{(\sqrt{2 N}+8 \alpha)^{2}}{2 N} c^{2 \gamma},
$$

where $C_{1}$ is such that $(2.6),(2.9)$ and $(2.10)$ hold. This together with (2.8) and (2.12) proves the lemma.

Lemma 2.3. If $c>C_{2}, \psi(\tau)=2 k \pi, u(\tau)=-c^{2 \gamma-1}, u^{\prime}(\tau)=0$, with $\tau \in$ $[0, T]$, then there exists $t_{2} \in\left(\tau, \tau+2 \sqrt{N} c^{\gamma-1}\right)$ such that

$$
\psi\left(t_{2}\right)=2 k \pi+\pi / 2,
$$

and

$$
\frac{1}{16 N} c^{2 \gamma} \leq E\left(t_{2}\right) \leq \frac{(\sqrt{2 N}+8)^{2}}{2 N} c^{2 \gamma} \leq Q c^{2 \gamma}
$$

In particular $E\left(t_{2}\right)=c^{2 \gamma^{\prime}}$ with $\gamma^{\prime} \in[\gamma-(\ln 16 N) /(2 \ln c), \gamma+(\ln Q) /(2 \ln c)]$.

Proof. Using the same arguments leading to the proof of (2.7) in Lemma 2.2 we get the existence of $t_{2} \in\left[\tau, \tau+2 \sqrt{N} c^{\gamma-1}\right]$ satisfying $u\left(t_{2}\right)=0$. Since for $u<0$ sufficiently large $|g(u)| \leq(M+1)|u| \leq(M+1) c^{2 \gamma-1}$, on $\left[\tau, t_{2}\right]$ we have

$$
u^{\prime}(t) \leq\left(3 c+(M+1) c^{2 \gamma-1}\right) \frac{1}{N} t \leq \frac{4}{N} c(t-\tau) .
$$

Thus,

$$
u(t) \leq-c^{2 \gamma-1}+\frac{2}{N} c(t-\tau)^{2} .
$$

Hence, $t_{2}-\tau \geq \sqrt{N} c^{\gamma-1} / \sqrt{2}$, which leads to $u^{\prime}\left(t_{2}\right) \geq c^{\gamma} / 2 \sqrt{2 N}$, and

$$
E\left(t_{2}\right) \geq \frac{1}{16 N} c^{2 \gamma}
$$

On the other hand by replacing $t_{1}$ with $\tau$ in (2.11) and using the fact that $u^{\prime}(\tau)=0$, we obtain

$$
E\left(t_{2}\right) \leq \frac{(\sqrt{2 N}+8)^{2}}{2 N} c^{2 \gamma}
$$

Hence, the lemma is proven.

Lemma 2.4. There exists $C_{3}$ such that if $c>C_{3}, E\left(t_{2}\right)=c^{2 \gamma}$, and $\psi\left(t_{2}\right) \in$ $[2 k \pi+\pi / 2,2 k \pi+\pi)$ for some $t_{2} \in\left[N c^{\gamma-1} / 4 \sqrt{Q}, T\right]$, then there exists $t_{3} \in$ $\left(t_{2}, t_{2}+2 c^{\gamma-1}\right)$ such that $\psi\left(t_{3}\right)=2 k \pi+\pi$, and

$$
\frac{3}{4} c^{2 \gamma} \leq E\left(t_{3}\right) \leq 2 c^{2 \gamma} \text {. }
$$

In particular $E\left(t_{3}\right)=c^{2 \gamma^{\prime}}$ with $\gamma^{\prime} \in\left[\gamma+\left(\ln \frac{3}{4}\right) /(2 \ln c), \gamma+(\ln 2) /(2 \ln c)\right]$.

Proof. Without loss of generality we can assume that $u\left(t_{2}\right) \leq g^{-1}(4 c)$. For $t>t_{2}$ such that $u(t) \leq g^{-1}(4 c)$, and $\psi(s) \in[2 k \pi+\pi / 2,2 k \pi+\pi]$, for all 
$s \in\left(t_{2}, t\right)$, we have

$$
\begin{aligned}
E(t) & =E\left(t_{2}\right)+\int_{t_{2}}^{t}\left(-\frac{N-1}{s} u^{\prime}(s)+c \varphi(s)+p(s)\right) u^{\prime}(s) d s \\
& \leq c^{2 \gamma}+3 c u(t)
\end{aligned}
$$

Since

$$
G(u(t)) \leq(4 c) g^{-1}(4 c) \leq 16 c^{1+(1 /(1+\rho))}
$$

for $c$ sufficiently large, from (2.17) using (2.4) we see that there exists a positive constant $\bar{\kappa}$ independent of $(c, \gamma)$ such that $\left(u^{\prime}(t)\right)^{2} \leq \bar{\kappa}^{2} c^{2 \gamma}$. On the other hand

$$
\begin{aligned}
E(t) & \geq E\left(t_{2}\right)+\int_{t_{2}}^{t}\left(-\frac{4(N-1) \sqrt{Q} \bar{\kappa}}{c^{\gamma-1} N} c^{\gamma}\right) u^{\prime}(s) d s \\
& \geq c^{2 \gamma}-\frac{4(N-1) \sqrt{Q} \bar{\kappa}}{N} c u(t) \\
& \geq c^{2 \gamma}-\frac{4(N-1) \sqrt{Q} \bar{\kappa}}{N} c^{1+(1 /(1+\rho))} \geq \frac{3}{4} c^{2 \gamma},
\end{aligned}
$$

for $c$ sufficiently large (see (2.4)). From (2.18) and (2.19) we have

$$
\left(u^{\prime}(t)\right)^{2} \geq \frac{3}{4} c^{2 \gamma}
$$

Thus

$$
(4 c)^{1 /(1+\rho)} \geq u(t) \geq \frac{\sqrt{3}}{2} c^{\gamma}\left(t-t_{2}\right) .
$$

Hence, there exists $t^{*} \in\left(t_{2}, t_{2}+6 c^{(1 /(1+\rho))-\gamma}\right)$ such that

$$
u\left(t^{*}\right)=g^{-1}(4 c) \text { and } u^{\prime}\left(t^{*}\right) \geq \frac{\sqrt{3}}{2} c^{\gamma} .
$$

For $t>t^{*}$ with $u^{\prime}(s) \geq 0$ for all $s \in\left[t^{*}, t\right]$ we have

$$
u^{\prime \prime}(t)=-\frac{N-1}{t} u^{\prime}(t)+c \varphi(t)+p(t)-g(u(t)) \leq-c .
$$

Hence,

$$
u^{\prime}(t) \leq u^{\prime}\left(t^{*}\right)-c\left(t-t^{*}\right) \leq \frac{\sqrt{3}}{2} c^{\gamma}-c\left(t-t^{*}\right) .
$$

Thus, there exists $t_{3} \in\left(t^{*}, t^{*}+\sqrt{3} c^{\gamma-1} / 2\right) \subset\left(t_{2}, t_{2}+2 c^{\gamma-1}\right),($ see $(2.4))$, such that

$$
u^{\prime}\left(t_{3}\right)=0
$$

for $c$ sufficiently large, and $u^{\prime}(t)>0$ for all $t \in\left[t^{*}, t_{3}\right)$. In particular

$$
\psi\left(t_{3}\right)=2 k \pi+\pi \text {. }
$$

Moreover, for $t \in\left[t_{2}, t_{3}\right]$ from (2.17) we have

$$
G(u(t)) \leq E(t) \leq c^{2 \gamma}+3 c u(t) .
$$


Thus from (1.4) we infer

$$
(u(t))^{2+\rho}-3 c u(t) \leq c^{2 \gamma} .
$$

Now, if $(u(t))^{2+\rho}<6 c u(t)$, then

$$
u(t)<6 c^{1 /(1+\rho)} .
$$

For $(u(t))^{2+\rho} \geq 6 c u(t)$ from (2.26) we see that

$$
u(t) \leq \frac{1}{3} c^{2 \gamma-1}
$$

By using (2.4), (2.27), and (2.28) for $c$ sufficiently large we infer

$$
u(t) \leq \frac{1}{3} c^{2 \gamma-1}
$$

Thus, replacing (2.29) in (2.25), for $t \in\left[t_{2}, t_{3}\right]$ we obtain

$$
E(t) \leq 2 c^{2 \gamma} \text {. }
$$

Hence, from (2.30) we have

$$
u^{\prime}(t) \leq c^{\gamma}
$$

Imitating the arguments leading to (2.19) it is easy to see that

$$
E\left(t_{3}\right) \geq \frac{3}{4} c^{2 \gamma}
$$

for $c>C_{3}$, where $C_{4}$ is assumed to be such that (2.19), (2.23), (2.29) and (2.32) hold. Thus, (2.24), (2.30) and (2.32) prove the lemma.

Lemma 2.5. There exists $C_{4}$ such that if $c>C_{4}, E\left(t_{3}\right)=c^{2 \gamma}$, and $\psi\left(t_{3}\right) \in$ $\left[2 k \pi+\pi, 2 k \pi+\frac{3}{2} \pi\right]$ for some $t_{3} \in\left[N c^{\gamma-1} / 8 \sqrt{Q}, T\right]$ then there exists $t_{4} \in$ $\left[t_{3}, t_{3}+2 c^{-\varepsilon / 2}\right]$ such that

Moreover,

$$
\psi\left(t_{4}\right)=2 k \pi+\frac{3}{2} \pi
$$

$$
\frac{3}{4} c^{2 \gamma} \leq E\left(t_{4}\right) \leq c^{2 \gamma} \text {. }
$$

In particular $E\left(t_{4}\right)=c^{2 \gamma^{\prime}}$ with $\gamma^{\prime} \in\left[\gamma+\left(\ln \frac{3}{4}\right) /(2 \ln c), \gamma\right]$.

Proof. Since $\psi\left(t_{3}\right) \in\left[2 k \pi+\pi, 2 k \pi+\frac{3}{2} \pi\right]$, we have $u\left(t_{3}\right)>0$ and $u^{\prime}\left(t_{3}\right) \leq 0$.

Hence either

$$
u\left(t_{3}\right)>g^{-1}\left(2 c+\|p\|_{\infty}+c^{\gamma+\varepsilon}\right)
$$

or

$$
u\left(t_{3}\right) \leq g^{-1}\left(2 c+\|p\|_{\infty}+c^{\gamma+\varepsilon}\right) .
$$

If (2.33) holds then for $t \geq t_{3}$ with $u(s) \geq g^{-1}\left(2 c+\|p\|_{\infty}+c^{\gamma+\varepsilon}\right)$ for all $s \in\left[t_{3}, t\right)$ we have

$$
\begin{aligned}
u^{\prime}(t) & =t^{-N+1}\left[t_{3}^{N-1} u^{\prime}\left(t_{3}\right)+\int_{t_{3}}^{t} s^{N-1}(c \varphi(s)+p(s)-g(u(s))) d s\right] \\
& \leq t^{-N+1} \int_{t_{3}}^{t} s^{N-1}\left(-c^{\gamma+\varepsilon}\right) d s \\
& =\frac{-c^{\gamma+\varepsilon}}{N}-\left[t-\left(t_{3}^{N}\right) / t^{N-1}\right] \leq 0 .
\end{aligned}
$$


Suppose that for all $t \in\left(t_{3}, t_{3}\left(1+c^{-\varepsilon / 2}\right)\right)$ we have $u(t)>g^{-1}\left(2 c+\|p\|_{\infty}+c^{\gamma+\varepsilon}\right)$. Hence, now for all $\lambda \in(-\varepsilon,-\varepsilon / 2)$, by $(2.35)$ we infer

$$
\begin{aligned}
u^{\prime}\left(t_{3}\left(1+c^{\lambda}\right)\right) & \leq \frac{-c^{\gamma+\varepsilon}}{N} t_{3}\left[\left(1+c^{\lambda}\right)-\left(1+c^{\lambda}\right)^{-N+1}\right] \\
& \leq \frac{-c^{\gamma+\varepsilon}}{2^{N-1}} \frac{N}{8 \sqrt{Q}} c^{\gamma-1} c^{\lambda} \leq-\frac{N}{2^{N+2} \sqrt{Q}} c^{2 \gamma-1} .
\end{aligned}
$$

Thus

$$
\begin{aligned}
u\left(t_{3}\left(1+c^{-\varepsilon / 2}\right)\right) & \leq u\left(t_{3}\left(1+c^{-\varepsilon}\right)\right)+\int_{t_{3}\left(1+c^{-\varepsilon}\right)}^{t_{3}\left(1+c^{-\varepsilon / 2}\right)}-c^{2 \gamma-1}\left[N /\left(2^{N+2} \sqrt{Q}\right)\right] d s \\
& \leq u\left(t_{3}\right)-\frac{N^{2}}{2^{N+5} Q} c^{3 \gamma-2}\left(c^{-\varepsilon / 2}-c^{-\varepsilon}\right) .
\end{aligned}
$$

Since $E\left(t_{3}\right)=c^{2 \gamma}$, we have that $u\left(t_{3}\right) \leq G^{-1}\left(c^{2 \gamma}\right)$. By (1.4) we see that for $c$ sufficiently large $g^{-1}\left(c^{2 \gamma}\right)<c^{2 \gamma /(1+\rho)}$. Hence, there exists $C_{4} \geq\|p\|_{\infty}$ such that for $c>C_{4}$

$$
u\left(t_{3}\right) \leq c^{2 \gamma /(2+\rho)} .
$$

Also, since $\varepsilon>0$ and (2.4) holds, we can assume $C_{4}$ to be such that $c^{-\varepsilon / 2}-$ $c^{-\varepsilon}>c^{-\varepsilon / 2} / 2$ for $c>C_{4}$, and that there exists a constant $k_{1}>0$ such that for $c>C_{4}$

$$
u\left(t_{3}\left(1+c^{-\varepsilon / 2}\right)\right) \leq c^{2 \gamma /(2+\rho)}-k_{1} c^{3 \gamma-2-(\varepsilon / 2)} \leq 0,
$$

which is a contradiction. Thus for $c>C_{4}$ there exists $t^{\prime} \in\left(t_{3}, t_{3}\left(1+c^{-\varepsilon / 2}\right)\right)$ such that

$$
u\left(t^{\prime}\right)=g^{-1}\left(2 c+\|p\|_{\infty}+c^{\gamma+\varepsilon}\right) .
$$

This and (2.34) show that there exists $t^{\prime \prime} \in\left[t_{3}, t_{3}\left(1+c^{-\varepsilon / 2}\right)\right]$ with

$$
u\left(t^{\prime \prime}\right) \leq g^{-1}\left(2 c+\|p\|_{\infty}+c^{\gamma+\varepsilon}\right) .
$$

Now we estimate $E(t)$ for $t>t_{3}$ with $u^{\prime}(s) \leq 0$ for all $s \in\left[t_{3}, t\right]$. Since

$$
\begin{aligned}
\frac{d E(t)}{d t} & =-\frac{N-1}{t}\left(u^{\prime}(t)\right)^{2}+(c \varphi(t)+p(t)) u^{\prime}(t) \\
& \leq\left(c-\|p\|_{\infty}\right) u^{\prime}(t) \leq 0,
\end{aligned}
$$

we have

$$
E(t) \leq c^{2 \gamma}
$$

Therefore,

$$
\left|u^{\prime}(t)\right| \leq \sqrt{2} c^{\gamma}
$$

Hence,

$$
-\frac{N-1}{t} u^{\prime}(t)+c \varphi(t)+p(t) \leq 4 c,
$$


for $c$ sufficiently large. If in addition $u(t) \geq 0$ then

$$
\begin{aligned}
E(t) & =E\left(t_{3}\right)+\int_{t_{3}}^{t}\left(-\frac{N-1}{t} u^{\prime}(s)+c \varphi(s)+p(s)\right) u^{\prime}(s) d s \\
& \geq c^{2 \gamma}+4 c\left(u(t)-u\left(t_{3}\right)\right) \geq c^{2 \gamma}-4 c u\left(t_{3}\right) \\
& \geq c^{2 \gamma}-4 c^{1+(2 \gamma /(2+\rho))} \geq \frac{3}{4} c^{2 \gamma}
\end{aligned}
$$

where we have used (2.4) and (2.39). Thus from (2.43) and (2.44) we obtain

$$
\frac{3}{4} c^{2 \gamma} \leq E\left(t_{2}\right) \leq c^{2 \gamma} \text {. }
$$

Now, for $t>t^{\prime \prime}$ we have

$$
\begin{aligned}
G(u(t)) & \leq u g(u) \leq g^{-1}(4 c) 4 c \\
& \leq 16 c^{1+(1 /(1+\rho))} \leq \frac{1}{4} c^{2 \gamma}
\end{aligned}
$$

(see (2.4)). Combining (2.47) and (2.48) we get

$$
u^{\prime}(t)<-c^{\gamma}
$$

for all $t \geq t^{\prime \prime}$ such that $u(s) \geq 0$ and $u^{\prime}(s) \leq 0$ with $s \in\left[t^{\prime \prime}, t\right]$. Hence

$$
\begin{aligned}
u(t) & \leq g^{-1}\left(2 c+\|p\|_{\infty}+c^{\gamma+\varepsilon}\right)-c^{\gamma}\left(t-t^{\prime \prime}\right) \\
& \leq 4 c^{1 /(1+\rho)}-c^{\gamma}\left(t-t^{\prime \prime}\right),
\end{aligned}
$$

(see (2.4)) for $c$ sufficiently large. From (2.50) we see that for some $t_{4} \in$ $\left[t^{\prime \prime}, t^{\prime \prime}+c^{(1 /(1+\rho))-\gamma}\right] \subset\left[t_{3}, t_{3}+2 c^{-\varepsilon / 2}\right]($ see $(2.4))$ we have

$$
u\left(t_{4}\right)=0
$$

in particular $\psi\left(t_{4}\right)=2 k \pi+\frac{3}{2} \pi$ for $c>C_{4}$, where $C_{4}$ is in addition assumed to be such that $(2.46)-(2.50)$ hold. This and (2.47) prove the lemma.

Lemma 2.6. There exists $C_{5}$ such that if $c>C_{5}, \psi\left(t_{4}\right) \in\left[2 k \pi+\frac{3}{2} \pi, 2(k+1) \pi\right]$, $E\left(t_{4}\right)=c^{2 \gamma}$, with $t_{4} \in\left[N c^{\gamma-1} / 8 \sqrt{Q}, T\right]$, and $u\left(t_{4}\right)>-\alpha c^{2 \gamma-1}, \alpha \in[1,64 N]$, then for some $t_{5} \in\left[t_{4}, t_{4}+\sqrt{2} c^{\gamma-1}\right]$ we have $\psi\left(t_{5}\right)=2(k+1) \pi$. Moreover,

$$
-(\alpha+4) c^{2 \gamma-1} \leq u\left(t_{5}\right) \leq-\frac{1}{64 \sqrt{Q}+16} c^{2 \gamma-1} .
$$

Proof. Let now $t>t_{4}$ be such that $u^{\prime}(s) \leq 0$ for all $s \in\left(t_{4}, t\right)$. The existence of such $t$ 's follows from the fact that $\psi\left(t_{4}\right) \in\left[2 k \pi+\frac{3}{2} \pi, 2(k+1) \pi\right]$. Since

$$
u^{\prime \prime}(s)=-\frac{N-1}{s} u^{\prime}(s)+c \varphi(s)+p(s)-g(u(s)) \geq \frac{c}{2}
$$

we have

$$
\begin{aligned}
u^{\prime}(t) & \geq u^{\prime}\left(t_{4}\right)+\frac{c}{2}\left(t-t_{4}\right) \geq-\sqrt{2 E\left(t_{4}\right)}+\frac{c}{2}\left(t-t_{4}\right) \\
& \geq-\sqrt{2} c^{\gamma}+\frac{c}{2}\left(t-t_{4}\right)
\end{aligned}
$$


Therefore, there exists $t_{5} \in\left[t_{4}, t_{4}+2 \sqrt{2} c^{\gamma-1}\right]$ with $u^{\prime}\left(t_{5}\right)=0$ and $u^{\prime}(s) \leq 0$ for all $s \in\left[t_{4}, t_{5}\right]$. Thus

$$
\psi\left(t_{5}\right)=2(k+1) \pi,
$$

and $\psi(s) \in\left[2 k \pi+\frac{3}{2} \pi, 2(k+1) \pi\right]$, for all $s \in\left[t_{4}, t_{5}\right]$. Furthermore,

$$
\begin{aligned}
u\left(t_{5}\right) & =u\left(t_{4}\right)+\int_{t_{4}}^{t_{5}} u^{\prime}(s) d s \geq-\alpha c^{2 \gamma-1}-\sqrt{2} c^{\gamma} 2 \sqrt{2} c^{\gamma-1} \\
& \geq-(\alpha+4) c^{2 \gamma-1} .
\end{aligned}
$$

By (1.3) there exist constants $M_{1}>0$, and $M_{2}$ such that $G(u) \leq M_{1} u^{2}+M_{2}$ for $u \leq 0$. Let $C_{5}$ be such that for $c>C_{5}$ we have

$$
G\left(-(\alpha+4) c^{2 \gamma-1}\right) \leq M_{1}(\alpha+4)^{2} c^{4 \gamma-2}+M_{2} \leq \frac{c^{2 \gamma}}{2} .
$$

Thus

$$
G\left(u\left(t_{4}\right)\right) \leq G\left(u\left(t_{5}\right)\right) \leq M_{1}\left(u\left(t_{5}\right)\right)^{2}+M_{2} \leq \frac{c^{2 \gamma}}{2} .
$$

Since $E\left(t_{4}\right)=c^{2 \gamma}$, from (2.56) we have

$$
u^{\prime}\left(t_{4}\right) \leq-c^{\gamma},
$$

for $c$ sufficiently large. By the continuity of $u^{\prime}$ we see that there exists $\tilde{t} \in$ $\left(t_{4}, t_{5}\right)$ such that

$$
u^{\prime}(\tilde{t})=-\frac{c^{\gamma}}{2} .
$$

Since $\left|u^{\prime}\left(t_{4}\right)\right| \leq \sqrt{2} c^{\gamma}$, and $|g(u)| \leq(M+1)|u|$ for $u \leq 0$ sufficiently large, then for $t \in\left[t_{4}, \tilde{t}\right]$ we have

$$
u^{\prime \prime}(t) \leq \frac{8(N-1) \sqrt{Q}}{c^{\gamma-1} N} \sqrt{2} c^{\gamma}+3 c+(M+1)(\alpha+4) c^{2 \gamma-1} \leq(16 \sqrt{Q}+4) c,
$$

for $c$ sufficiently large. Thus by integrating (2.59) on $\left[t_{4}, \tilde{t}\right]$, and using (2.57) and (2.58) we have that $\tilde{t}-t_{4} \geq c^{\gamma-1} / 2(16 \sqrt{Q}+4)$. Hence

$$
\begin{aligned}
u\left(t_{5}\right) & \leq u(\tilde{t})=u\left(t_{4}\right)+\int_{t_{4}}^{\tilde{t}} u^{\prime}(s) d s \\
& \leq 0-\frac{c^{\gamma}}{2}\left(\tilde{t}-t_{4}\right) \leq-\frac{c^{2 \gamma-1}}{64 \sqrt{Q}+16} .
\end{aligned}
$$

From (2.54) and (2.60) we see that if $c>C_{5}$, where $C_{5}$ is in addition assumed to be such that $(2.59)$ holds, then the lemma is proven.

The next lemma summarizes the results of this section. 
Lemma 2.7. Given any positive integer $j$ there exists a real number $m_{j}$ such that if $c>m_{j}, E(t, d, c)>0$ for all $t \in[0, T], E\left(t_{1}, d, c\right)=c^{2 \gamma}$, for some $t_{1} \in\left[N c^{\gamma-1} / 4, T\right], \gamma \in\left[\left(5 \gamma_{1}+3 \gamma_{2}\right) / 8,\left(3 \gamma_{1}+5 \gamma_{2}\right) / 8\right]$, and either

(A) $\psi\left(t_{1}, d, c\right) \in\left[2 k \pi+\frac{\pi}{2}, 2 k \pi+\frac{3}{2} \pi\right]$, or

(B) $\psi\left(t_{1}, d, c\right) \in\left[2 k \pi, 2 k \pi+\frac{\pi}{2}\right) \cup\left(2 k \pi+\frac{3}{2} \pi, 2(k+1) \pi\right]$, and $u\left(t_{1}, d, c\right) \geq$ $-c^{2 \gamma-1}$,

then $\psi(T, d, c)>j \pi+\pi / 2$.

Proof. We split the proof into four cases depending on the quadrant where $\psi\left(t_{1}, d, c\right)$ lies.

Case I. Suppose that $\psi\left(t_{1}, d, c\right) \in[2 k \pi, 2 k \pi+\pi / 2]$. By (2.4) there exists $C_{5}$ such that for $c>C_{5}$ we have that $(2 \sqrt{2}+2 \alpha \sqrt{N}+2) c^{\gamma_{2}-1}<c^{-\varepsilon / 2}$. Thus by applying consecutively Lemmas 2.2 (or 2.3), 2.4, 2.5, 2.6, we see that if $c>C:=\max \left\{C_{1}, \ldots, C_{5}\right\}$ then there exist $t_{2}<t_{3}<t_{4}<t_{5}$ such that

$$
t_{5} \in\left[t_{1}, t_{1}+k^{*} c^{-(\varepsilon / 2)}\right]
$$

and

$$
\psi\left(t_{5}, d, c\right)=2(k+1) \pi .
$$

Moreover, from Lemma 2.2. we have

$$
E\left(t_{2}, d, c\right)=c^{2 \gamma^{\prime}},
$$

with

$$
-\frac{\ln 128}{2 \ln c}+\gamma \leq \gamma^{\prime} \leq \gamma+\frac{\ln Q}{2 \ln c} .
$$

Also, by combining (2.64) with the results from Lemmas 2.4 and 2.5 we see that $E\left(t_{4}, d, c\right)=c^{2 \gamma^{\prime \prime}}$, with

$$
\gamma-\frac{2 \ln (3 / 4)+\ln 128}{2 \ln c} \leq \gamma^{\prime \prime} \leq \gamma+\frac{\ln 2+\ln Q}{2 \ln c} .
$$

Now by Lemma 2.6 (since in this case $\alpha=0$ ) we have that $u\left(t_{5}\right)=-c^{2 \gamma-1}$ with

$$
\gamma-\frac{\ln (72(64 \sqrt{Q}+16))}{2 \ln c} \leq \bar{\gamma} \leq \gamma+\frac{\ln 8 Q}{2 \ln c}
$$

or

$$
\begin{aligned}
-k^{\prime} c^{2 \gamma-1} & :=-8 Q c^{2 \gamma-1} \leq u\left(t_{5}\right) \leq-[72(64 \sqrt{Q}+16)] c^{2 \gamma-1} \\
& :=-K^{\prime} c^{2 \gamma-1}
\end{aligned}
$$

Let now

$$
m_{j}:=\max \left\{\exp \left(\frac{4 j\left|\ln k^{\prime}\right|}{3\left(\gamma_{2}-\gamma_{1}\right)}\right), \exp \left(\frac{4 j\left|\ln K^{\prime}\right|}{3\left(\gamma_{2}-\gamma_{1}\right)}\right),(j / T)^{2 / \varepsilon}, C\right\} .
$$


From (2.67) and (2.68) it follows that

$$
\frac{5 \gamma_{1}+3 \gamma_{2}}{8}-\frac{3 \gamma_{2}-3 \gamma_{1}}{8 j} \leq \bar{\gamma} \leq \frac{3 \gamma_{1}+5 \gamma_{2}}{8}+\frac{3 \gamma_{2}-3 \gamma_{1}}{8 j}
$$

Also, from (2.68) we have

$$
t_{5}-t_{1} \leq T / j
$$

Now, we observe that $t_{5}=\tau$, and $\bar{\gamma}=\gamma$ satisfy the hypothesis of Lemma 2.3. Thus, iterating the above argument $j$ times we see that there exist $t_{5}<t_{6}<$ $\cdots<t_{j+3}<T$, with $\psi\left(t_{j+3}, d, c\right)=2(k+j) \pi$. Hence, by Remark 2.1. we have that

$$
\psi(T, d, c)>2(k+j) \pi-\frac{\pi}{2} \geq 2 j \pi-\frac{\pi}{2} \geq j \pi+\frac{\pi}{2},
$$

and that proves Case I.

Case II. If $\psi\left(t_{1}, d, c\right) \in[2 k \pi+\pi / 2,(2 k+1) \pi]$, then by applying Lemmas 2.4 , 2.5, 2.6. we see that there exists $t_{5}$ that satisfies $(2.61),(2.62),(2.67),(2.69)$, and thus, we are in Case I.

Case III. If $\psi\left(t_{1}, d, c\right) \in[(2 k+1) \pi,(2 k+1) \pi+\pi / 2]$, then we can apply Lemmas 2.5 and 2.6, and Case III reduces to Case I.

Case IV. If $\psi\left(t_{1}, d, c\right) \in[(2 k+1) \pi+\pi / 2,2(k+1) \pi]$, then by applying Lemma 2.6 we are in Case I, and that concludes the proof of Lemma 2.7.

\section{ENERGY ANALYSIS}

Throughout this section we use $K, k^{\prime}, K^{\prime}, \bar{k}, k_{1}, k_{2}$ to denote various constants independent of $(c, \gamma)$.

Let now $u(t):=u(t, d, c)$ be a solution to (1.8). Suppose that there exists $\hat{t} \in\left[N c^{\gamma-1} / 4, T\right]$, with $\gamma \in\left[\gamma_{1}, \gamma_{2}\right]$, such that

$$
E(\hat{t}, d, c)=c^{2 \gamma}, \quad E(t)>0 \text { for all } t \in[0, \hat{t}] \text { and } u(\hat{t}) \geq-c^{2 \gamma-1} .
$$

Arguing as in the proof of Lemma 2.7 (using Lemmas 2.2, 2.4 and 2.5 if $\psi(\hat{t}, d, c) \in[2 k \pi, 2 k \pi+\pi / 2]$, Lemmas 2.4 and 2.5 if $\psi(\hat{t}, d, c) \in[2 k \pi+$ $\pi / 2,(2 k+1) \pi]$, Lemma 2.5 if $\psi(\hat{t}, d, c) \in[(2 k+1) \pi,(2 k+1) \pi+\pi / 2]$, and Lemmas $2.6,2.3,2.4$ and 2.5 if $\psi(\hat{t}, d, c) \in[(2 k+1) \pi+\pi / 2,2(k+1) \pi])$, we see that there exists $\tau_{1} \in\left[\hat{t}, \hat{t}+K c^{-(\varepsilon / 2)}\right]$ such that $u\left(\tau_{1}\right)=0, u^{\prime}\left(\tau_{1}\right)<0$, and

$$
k^{\prime} c^{2 \gamma} \leq E\left(\tau_{1}, d, c\right) \leq K^{\prime} c^{2 \gamma} \text {. }
$$

Now we prove by induction

Lemma 3.1. Let $\hat{t}$ be as in (3.1) and let $\hat{t} \leq \tau_{1}<\hat{\tau}_{1}<\tau_{2}<\cdots<\tau_{i}<\hat{\tau}_{i}<$ $\tau_{i+1}<\cdots \leq t$ denote the zeroes of $u(t)$ for $t>\tau_{1}$ such that $E(s, d, c)>0$ for $s \in\left[\tau_{1}, t\right)$. If for some $\kappa \in(0,1) L(\kappa)=\infty$, then there exists $c^{*}$ such that if $c>c^{*}$ then

$$
\begin{aligned}
k^{\prime} c^{2 \gamma+(\gamma-1) N} & \leq \tau_{i}^{N} E\left(\tau_{i}, d, c\right) \\
& \leq \tau_{i}^{N}\left[k^{\prime \prime} c^{2 \gamma}+(i-1) c^{3 \gamma-1}\right] \leq K^{\prime} c^{2 \gamma} .
\end{aligned}
$$

In particular $E(t, d, c)>0$ for all $t \in[0, T]$. 
Proof. Since $\tau_{1} \geq \hat{t} \geq c^{\gamma-1}$ from (3.2) we have (3.3) for $i=1$. Also, from (3.2) we see that

$$
k^{\prime} c^{2 \gamma+(\gamma-1) N} \leq E\left(\tau_{i}, d, c\right) \leq K^{\prime} c^{2 \gamma} .
$$

Thus, as in (2.54) we obtain the existence of $a \in\left[\tau_{i}, \tau_{i}+K c^{\gamma-1}\right]$ such that $u^{\prime}(a)=0$ and $u(a)>-(\alpha+4) c^{2 \gamma-1}$. Furthermore, since $u^{\prime \prime}(t) \leq K c^{1-\gamma} c^{\gamma}+$ $3 c+(M+1)|u(a)| \leq K c$ for $t \in\left[\tau_{i}, a\right]$ we infer $0=u^{\prime}(a) \leq-c^{\gamma+(\gamma-1)(N / 2)}+$ $K c\left(a-\tau_{i}\right)$. Therefore $a-\tau_{i} \geq K c^{\gamma-1+(\gamma-1)(N / 2)}$.

Also, by the continuity of $u^{\prime}$ we see that there exists $\hat{a} \in\left[\tau_{i}, a\right]$ such that $u^{\prime}(\hat{a})=\frac{1}{2} u^{\prime}\left(\tau_{i}\right)$. Since $u^{\prime}(\hat{a})=u^{\prime}\left(\tau_{i}\right)+\int_{\tau_{i}}^{\hat{a}} u^{\prime \prime}(s) d s \leq u^{\prime}\left(\tau_{i}\right)+K c\left(\hat{a}-\tau_{i}\right)$ we have $\hat{a}-\tau_{i} \geq K c^{\gamma-1+(\gamma-1)(N / 2)}$. Thus

$$
u(a) \leq u(\hat{a})=u\left(\tau_{i}\right)+u^{\prime}(\hat{a})\left(\hat{a}-\tau_{i}\right) \leq-K c^{2 \gamma-1+(\gamma-1) N} .
$$

Arguing as in (2.6)-(2.7) we see that $\hat{\tau}_{i}<a+K c^{\gamma-1}$. In particular there exists a unique $b\left(u\right.$ is convex on $\left.\left(\tau_{i}, \hat{\tau}_{i}\right)\right)$ such that $u(b)=-c^{1 /(1+\rho)}$. Since

$$
u(b)=u(a)+\int_{a}^{b} u^{\prime}(s) d s \leq-K c^{2 \gamma-1+(\gamma-1) N}+(b-a) K c^{\gamma},
$$

we infer

$$
b-a \geq K c^{\gamma-1+(\gamma-1) N},
$$

where we have also used the fact that $2 \gamma+(\gamma-1) N-1>1 /(1+\rho)$. Hence, as in (2.12) we obtain that $E\left(\hat{\tau}_{i}, d, c\right) \geq K c^{2 \gamma+(\gamma-1) N}$. Imitating the arguments used in Lemma 2.4 and Lemma 2.5 we show the existence of $\hat{\tau}_{i}<\tau_{i}^{\prime}<\tau_{i}^{*}<\tau_{i}^{\prime \prime}<\tau_{i+1}$ such that $u\left(\tau_{i}^{\prime}\right)=u\left(\tau_{i}^{\prime \prime}\right)=K g^{-1}(4 c), u^{\prime}\left(\tau_{i}^{\prime}\right)>0, u^{\prime}\left(\tau_{i}^{\prime \prime}\right)<0$, and $u^{\prime}\left(\tau_{i}^{*}\right)=0$. In addition, we obtain that

$$
\tau_{i}^{\prime}-\tau_{i} \leq K c^{[1 /(1+\rho)]-\gamma-(\gamma-1)(N / 2)},
$$

and $E\left(\tau_{i}^{*}, d, c\right) \geq K c^{2 \gamma+(\gamma-1) N}$. Therefore, as in Lemma 2.4 we infer

$$
\tau_{i+1}-\tau_{i}^{\prime \prime} \leq K c^{[1 /(1+\rho)]-\gamma-(\gamma-1)(N / 2)} .
$$

Since $[1 /(1+\rho)]-\gamma-(\gamma-1)(N / 2)<\gamma+(\gamma-1) N-1$ (see $(2.4 \mathrm{a}))$ we have

$$
\left|\int_{a}^{b} K c r^{N-1} u(r) d r\right|>\left|\int_{\left[\tau_{i}, \tau_{i}^{\prime}\right] \cup\left[\tau_{i}^{\prime \prime}, \tau_{i+1}\right]} K c r^{N-1} u(r) d r\right| .
$$

Multiplying (1.8) by $r^{N} u^{\prime}$ and integrating over $\left[\tau_{i}, \tau_{i+1}\right]$ we obtain

$$
\begin{aligned}
\tau_{i+1}^{N} E\left(\tau_{i+1}, d, c\right)-\tau_{i}^{N} E\left(\tau_{i}, d, c\right) \\
\quad+\int_{\tau_{i}}^{\tau_{i+1}}\left[\frac{N-2}{2} r^{N-1}\left(u^{\prime}(r)\right)^{2}-N r^{N-1} G(u(r))\right] d r \\
=\int_{\tau_{i}}^{\tau_{i+1}}[c \varphi(r)+p(r)] r^{N} u^{\prime}(r) d r,
\end{aligned}
$$


where we have integrated by parts the term $\int_{\tau_{i}}^{\tau_{i+1}} r^{N}\left[u^{\prime \prime}(r) u^{\prime}(r)+(G(u(r)))^{\prime}\right] d r$. Similarly, multiplying (1.8) by $r^{N-1} u$ and integrating over $\left[\tau_{i}, \tau_{i+1}\right]$ we infer

$$
\begin{aligned}
\int_{\tau_{i}}^{\tau_{i+1}} & r^{N-1}\left(u^{\prime}(r)\right)^{2} d r \\
\quad= & \int_{\tau_{i}}^{\tau_{i+1}} r^{N-1}\{g(u(r)) u(r)-[c \varphi(r)+p(r)] u(r)\} d r .
\end{aligned}
$$

By replacing (3.10) in (3.9) we obtain

$$
\begin{aligned}
\tau_{i+1}^{N} E\left(\tau_{i+1}, d, c\right)-\tau_{i}^{N} E\left(\tau_{i}, d, c\right) \\
\left.=\int_{\tau_{i}}^{\tau_{i+1}} r^{N-1}\left[N G(u(r))-\frac{N-2}{2} g(u(r)) u(r)\right)\right] d r \\
\quad+\int_{\tau_{i}}^{\tau_{i+1}} r^{N-1}[c \varphi(r)+p(r)]\left[r u^{\prime}(r)+\frac{N-2}{2} u(r)\right] d r .
\end{aligned}
$$

Integrating by parts the last term we get

$$
\begin{aligned}
& \tau_{i+1}^{N} E\left(\tau_{i+1}, d, c\right)-\tau_{i}^{N} E\left(\tau_{i}, d, c\right) \\
& \geq \int_{\tau_{i}}^{\tau_{i+1}} r^{N-1}\left[N G(u(r))-\frac{N-2}{2} g(u(r)) u(r)\right] d r \\
&-\frac{c}{2} \int_{\tau_{i}}^{\tau_{i+1}}\left(\frac{N}{2}+1\right) r^{N-1} u(r) d r \\
& \geq \int_{\left[\tau_{i}, \tau_{i}^{\prime}\right] \cup\left[\tau_{\left.\tau_{i}^{\prime \prime}, \tau_{i+1}\right]}\right.} r^{N-1}\left[N G(u(r))-\frac{N-2}{2} g(u(r)) u(r)-\bar{k} c u(r)\right] d r \\
&+\int_{\tau_{i}^{\prime}}^{\tau_{i}^{\prime \prime}} r^{N-1}\left[N G(u(r))-\frac{N-2}{2} g(u(r)) u(r)-\bar{k} c u(r)\right] d r \\
&-\bar{k} c \int_{a}^{b} r^{N-1} u(r) d r .
\end{aligned}
$$

Since $g$ is an increasing function for $u>0$ we have

$$
G(u)=\int_{0}^{\kappa u} g(s) d s+\int_{\kappa u}^{u} g(s) d s \geq G(\kappa u)+(1-\kappa) u g(\kappa u) .
$$

Thus, from the assumption that $L(\kappa)=\infty$, there exists $k_{1} \geq 0$ such that for $u \geq k_{1} \quad\left(k_{1}\right.$ is chosen so that $N G(\kappa u)-((N-2) / 2) g(u) u \geq 0$ for $\left.\kappa u \geq k_{1}\right)$

$$
\begin{aligned}
N G(u)-\frac{N-2}{2} g(u) u & \geq N G(\kappa u)+N\left(\frac{1-\kappa}{\kappa}\right) \kappa u g(\kappa u)-\frac{N-2}{2} g(u) u \\
& \geq N\left(\frac{1-\kappa}{\kappa}\right) \kappa u g(\kappa u) \geq N\left(\frac{1-\kappa}{\kappa}\right) G(\kappa u) \\
& \geq\left(\frac{1-\kappa}{\kappa}\right) \frac{N-2}{2} g(u) u=k_{2} g(u) u
\end{aligned}
$$


Thus if $c$ is large enough and $\tau_{i}^{\prime}, \tau_{i}^{\prime \prime}$ are chosen so that $g\left(u\left(\tau_{i}^{\prime}\right)\right)=g\left(u\left(\tau_{i}^{\prime \prime}\right)\right)=$ $\overline{k c} / k_{2}$ then

$$
\int_{\tau_{i}^{\prime}}^{\tau_{i}^{\prime \prime}} r^{N-1}\left[N G(u(r))-\frac{N-2}{2} g(u(r)) u(r)-c \bar{k} u(r)\right] d r \geq 0 .
$$

From (1.3) it follows that $L(1, u)$ is bounded below. Thus, (3.8), (3.14) and (3.12) yield

$$
\tau_{i+1}^{N} E\left(\tau_{i+1}, d, c\right) \geq \tau_{i}^{N} E\left(\tau_{i}, d, c\right) \geq K c^{2 \gamma+(\gamma-1) N} .
$$

On the other hand, since $\varphi$ is differentiable we have

$$
\begin{aligned}
E\left(\tau_{i+1}\right) & \leq E\left(\tau_{i}\right)+\int_{\tau_{i}}^{\tau_{i+1}}(c \varphi(s)+p(s)) u^{\prime}(s) d s \\
& \leq E\left(\tau_{i}\right)-c \int_{\tau_{i}}^{\tau_{i+1}} \varphi^{\prime}(s) u(s) d s \leq E\left(\tau_{i}\right)+K c \int_{\tau_{i}}^{\hat{\tau}_{i}}|u(s)| d s \\
& \leq E\left(\tau_{i}\right)+K c c^{2 \gamma-1} c^{\gamma-1} .
\end{aligned}
$$

Hence inductively we obtain

$$
E\left(\tau_{i+1}\right) \leq E\left(\tau_{1}\right)+(i-1) K c^{3 \gamma-1} .
$$

Since $\tau_{i+1}-\tau_{i} \geq K c^{\gamma-1}$, we see that $i \leq\left(T /\left(K c^{\gamma-1}\right)\right)$. Therefore

$$
E\left(\tau_{i+1}\right) \leq E\left(\tau_{1}\right)+K c^{2 \gamma} \leq K^{\prime} c^{2 \gamma},
$$

which together with (3.15) proves (3.3).

Let $\gamma_{0}:=\left(\gamma_{1}+\gamma_{2}\right) / 2$, where $\gamma_{1}$ and $\gamma_{2}$ are as in (2.3).

Lemma 3.2. Let $\tau \in[0, T]$ be such that $u^{\prime}(\tau) \geq 0$ and $u(\tau) \leq-c^{2 \gamma_{0}-1}$. There exist $C$ such that if $c>C$ then for some

$$
\tau_{1} \in\left(\sqrt{2 N}\left(\frac{|u(\tau)|}{3 c+(M+1)|u(\tau)|}\right)^{(1 / 2)}, 2 \sqrt{N}\left(\frac{|u(\tau)|}{c}\right)^{(1 / 2)}\right)
$$

we have $u\left(\tau_{1}+\tau\right)=0, u(t)<0$ for all $t \in\left[\tau, \tau+\tau_{1}\right]$, and $E\left(\tau_{1}, d, c\right) \geq$ $c^{2 \gamma_{0}} / 64 N$.

Proof. Since $g$ is an increasing function, for $t>\tau$ we have

$$
u^{\prime}(t) \geq t^{-N+1} \frac{c}{2} \int_{\tau}^{t} s^{N-1} d s \geq \frac{c}{2 N}(t-\tau)
$$

Hence $u(t) \geq u(\tau)+(c / 4 N)(t-\tau)^{2}$. Therefore, $\tau_{1} \leq 2 \sqrt{N}(|u(\tau)| / c)^{(1 / 2)}$ exists such that $u\left(\tau_{1}+\tau\right)=0$. Similarly using that for $u<0$ sufficiently large $|g(u)| \leq(M+1)|u|$ we have $u^{\prime}(t)<t(3 c+(M+1)|u(\tau)|) / N$. Thus, $u(t) \leq$ $u(\tau)+t^{2}(3 c+(M+1)|u(\tau)|) /(2 N)$. This proves that

$$
\tau_{1} \geq \sqrt{2 N}\left(\frac{|u(\tau)|}{3 c+(M+1)|u(\tau)|}\right)^{(1 / 2)} \geq \frac{\sqrt{2 N}}{4} c^{\gamma_{0}-1}
$$


Hence,

$$
u^{\prime}\left(\tau_{1}\right) \geq \frac{c-\|p\|_{\infty}}{4 N} \sqrt{2 N} c^{\gamma_{0}-1} \geq \frac{1}{4 \sqrt{2 N}} c^{\gamma_{0}} .
$$

Therefore, $E\left(\tau_{1}, d, c\right) \geq c^{2 \gamma_{0}} / 64 N$, and that concludes the proof of the lemma.

Lemma 3.3. Let $C$ be as in Lemma 3.2, and $c>C$. If $\bar{t} \in[0, T]$ is the largest number such that $E(\bar{t}, d, c)=c^{2 \gamma_{0}} / 64 N$, and $E(t, d, c)>0$ for all $t \in[0, \bar{t}]$ then $u(\bar{t}) \geq-c^{2 \gamma_{0}-1}$.

Proof. Suppose that $u(\bar{t})<-c^{2 \gamma_{0}-1}$. From Lemma 3.2 for all $t>\bar{t}$ with $u(t)<0$ we have

$$
u^{\prime \prime}(t) \geq-\frac{4(N-1)}{\sqrt{2 N}} c^{1-\gamma_{0}} \frac{1}{4 \sqrt{2 N}} c^{\gamma_{0}}+c \varphi(t)+p(t)-g(u(t)) \geq \frac{c}{2}\left(1-\frac{N-1}{2 N}\right) .
$$

If $u^{\prime}(\bar{t})<0$ we let $t_{0}$ denote the smallest number greater than $\bar{t}$ such that $u^{\prime}\left(t_{0}\right)=0$, and if $u^{\prime}(\bar{t})>0$ we let $t_{0}=\bar{t}$. Of course, $u\left(t_{0}\right) \leq u(\bar{t})<-c^{2 \gamma_{0}-1}$. Since for all $s>t_{0}$ with $u<0$ on $\left(t_{0}, s\right)$ we have that $\left|u^{\prime}(s)\right| \leq c^{\gamma_{0}} / 4 \sqrt{2 N}$, hence $u(t)<0$ for $t \in\left(t_{0}, t_{0}+4 \sqrt{2 N} c^{\gamma_{0}-1}\right)$. Thus, for $t=t_{0}+4 \sqrt{2 N} c^{\gamma_{0}-1}$ we have

$$
\begin{aligned}
u^{\prime}\left(t_{0}+4 \sqrt{2 N} c^{\gamma_{0}-1}\right) & =u^{\prime}\left(t_{0}\right)+\int_{t_{0}}^{t_{0}+4 \sqrt{2 N} c^{\gamma_{0}-1}} u^{\prime \prime}(s) d s \\
& \geq \frac{c}{2}\left(1-\frac{N-1}{2 N}\right) 4 \sqrt{2 N} c^{\gamma_{0}-1}>\frac{1}{4 \sqrt{2 N}} c^{\gamma_{0}}
\end{aligned}
$$

which contradicts the assumption that $\bar{t}$ is the largest number such that $E(\bar{t}, d, c)=c^{2 \gamma_{0}} / 64 N$ and $E(t, d, c)>0$ for all $t \in[0, \bar{t}]$. Hence the lemma is proven.

Now we summarize the above results in the following lemma.

Lemma 3.4. If $L(\kappa)=\infty$ for some $\kappa \in(0,1)$, then there exists $C^{*}$ such that if $c>C^{*}$, and $d \leq-c^{2 \gamma_{0}-1}$ then $E(t, d, c)>0$ for all $t \in[0, T]$.

Proof. From Lemma 3.2 (taking $\tau=0$ ) we see that either $E(t, d, c) \geq c^{2 \gamma_{0}} / 64 N$ for all $t \in[0, T]$, or there exists $\bar{t} \geq \tau_{1}>\frac{1}{4} c^{2 \gamma_{0}-1}$ such that $E(\bar{t}, d, c)=$ $c^{2 \gamma_{0}} / 64 N$ and $E(t, d, c)>0$ for all $t \in[0, t]$. Also by Lemma 3.3 we have $u(\bar{t}) \geq-c^{2 \gamma_{0}-1}$. Hence, taking $\gamma^{\prime}=\gamma_{0}-(\ln 64 N) /(2 \ln c)$ we have $E(\bar{t}, d, c)=c^{2 \gamma^{\prime}}$, and

$$
u(\bar{t}) \geq-c^{2 \gamma_{0}-1}=-c^{2\left(\gamma_{0}-\gamma^{\prime}\right)} c^{2 \gamma^{\prime}-1}=-64 N c^{2 \gamma^{\prime}-1} \equiv-\alpha c^{2 \gamma^{\prime}-1} .
$$

Let now $\bar{c}$ be such that if $c>\bar{c}$ then $(\ln 64 N) /(2 \ln c)<\frac{1}{4}\left(\gamma_{2}-\gamma_{1}\right)$. By applying consecutively the lemmas from section 2 , depending on the quadrant where $u(\bar{t})$ lies we obtain the existence of $\tau_{1} \in\left[\bar{t}, \bar{t}+K c^{-(\varepsilon / 2)}\right]$ such that $u\left(\tau_{1}\right)=0, u^{\prime}\left(\tau_{1}\right)<0$ and (3.2) holds. Now, directly from Lemma 3.1 by taking $C^{*}=\max \left\{c^{*}, C, \bar{c}\right\}$ we obtain the proof of the lemma. 


\section{SeParation of Zeroes}

The following lemma is a version of the Sturm comparison theorem for singular linear differential equations, and it proves our main estimate on the separation of zeroes.

Lemma 4.1. There exists $H:=H(c)>0$ such that if $u$ satisfies (1.8), $u\left(t^{0}\right)<-H$ and $u^{\prime}\left(t^{0}\right)=0$, for some $t^{0} \in[0, T]$, then $u(t)<0$ for all $t \in$ $\left(t^{0}, t^{0}+\frac{1}{2}(M+1)^{-1 / 2}\right)$. Moreover, if for some $t^{0} \in[0, T], u^{\prime}\left(t^{0}, d, c\right)=0$ and $u\left(t^{0}, d, c\right)<-H$, then

$$
\psi(T, d, c) \leq \psi\left(t^{0}, d, c\right)+\pi / 2+2 J\left(t^{0}\right) \pi,
$$

where $J\left(t^{0}\right)$ is the greatest integer less then $2(M+1)^{1 / 2}\left(T-t^{0}\right)$. In particular taking $t^{0}=0$ we have

$$
\limsup _{-d \rightarrow \infty} \psi(T, d, c) \leq \frac{\pi}{2}+2 J(0):=S .
$$

Proof. From (1.3)-(1.4) we see that there exists a real number $M^{*} \leq 0$ such that

$$
g(s)-(M+1) s \geq M^{*} \text { for all } s \in \mathbf{R} .
$$

Thus, for $A(t):=p(t)+c \varphi(t)-g(u(t))+(M+1) u(t)$, we have

$$
A(t) \leq\|p\|_{\infty}+c\|\varphi\|_{\infty}-M^{*}:=H .
$$

By rewriting (1.8) we obtain

$$
\begin{gathered}
u^{\prime \prime}(t)+\frac{N-1}{t} u^{\prime}(t)+(M+1) u(t)=A(t), \\
u\left(t^{0}\right)=-h, \quad u^{\prime}\left(t^{0}\right)=0,
\end{gathered}
$$

where $h \in \mathbf{R}$ is such that $h>H$. Let now

$$
F(t)=\frac{1}{2}\left[\left(u^{\prime}(t)\right)^{2}+(M+1) u^{2}(t)\right] .
$$

From (4.3) and (4.4) we see that

$$
d F / d t \leq A(t) u^{\prime}(t) \leq H(2 F(t))^{1 / 2} .
$$

Integrating $(4.5)$ on $\left[t^{0}, t\right]$ we obtain

$$
\begin{aligned}
\left|u^{\prime}(t)\right| & \leq(2 F(t))^{1 / 2} \leq\left(2 F\left(t^{0}\right)\right)^{1 / 2}+H\left(t-t^{0}\right) \\
& \leq(M+1)^{1 / 2} h+H\left(t-t^{0}\right) .
\end{aligned}
$$

Let $\tau>t^{0}$ be such that $u(\tau)=0$, and $u(t)<0$ for all $t \in\left[t^{0}, \tau\right]$. Hence, from (4.6) we have

$$
\begin{aligned}
0 & =u(\tau)=u\left(t^{0}\right)+\int_{t^{0}}^{t} u^{\prime}(s) d s \\
& \leq u\left(t^{0}\right)+(M+1)^{1 / 2} h\left(t-t^{0}\right)+\frac{1}{2} H\left(t-t^{0}\right)^{2} \\
& \leq h\left[-1+(M+1)^{1 / 2}\left(t-t^{0}\right)+\frac{1}{2}\left(t-t^{0}\right)^{2}\right] .
\end{aligned}
$$


Therefore

$$
t-t^{0} \leq \frac{1}{2}(M+1)^{-1 / 2}:=\Lambda \text {. }
$$

Hence, $u(t)<0$ for all $t \in\left[t^{0}, t^{0}+\Lambda\right]$, and thus in particular

$$
\psi\left(t^{0}+\Lambda, d, c\right) \leq \psi\left(t^{0}, d, c\right)+\pi / 2 .
$$

Iterating this argument we see that

$$
\psi\left(t^{0}+2 \Lambda, d, c\right) \leq \psi\left(t^{0}, d, c\right)+\pi / 2+2 \pi,
$$

and

$$
\psi\left(t^{0}+i \Lambda, d, c\right) \leq \psi\left(t^{0}, d, c\right)+\pi / 2+(i-1) 2 \pi,
$$

with $i \in\left\{0,1, \ldots, J\left(t^{0}\right)\right\}$. In particular

$$
\begin{aligned}
\psi(T, d, c) & \leq \psi\left(t^{0}+J\left(t^{0}\right) \Lambda, d, c\right)+2 \pi \\
& \leq \psi\left(t^{0}, d, c\right)+\pi / 2+\left(J\left(t^{0}\right)-1\right) 2 \pi+2 \pi \\
& =\psi\left(t^{0}, d, c\right)+\pi / 2+J\left(t^{0}\right) 2 \pi,
\end{aligned}
$$

and that proves the lemma.

\section{Proof of Theorem A}

By Lemma 3.4 we see that there exists $C^{*}$ such that if $c>C^{*}$ and $d \leq$ $-c^{2 \gamma_{0}-1}$ then $E(t, d, c)>0$ for all $t \in[0, T]$. Therefore, if $c>m_{j}$ then by Lemma 2.7 we have that

$$
\psi\left(T,-c^{2 \gamma_{0}-1}, c\right) \geq j \pi+\pi / 2 .
$$

Let $J:=[(S-\pi / 2) / \pi]+1$, where $[x]$ denotes the largest integer less than or equal to $x$. If $j \geq J$ then $j \pi+\pi / 2>S$. Hence by Lemma 4.1, (5.1), and the intermediate value theorem if $c>C_{j}=\max \left\{m_{j}, C^{*}\right\}$ then there exist numbers $d_{J}<d_{J+1}<\cdots<d_{j}$, such that

$$
\psi\left(T, d_{i}, c\right)=i \pi+\pi / 2, \quad i \in\{J, J+1, \ldots, j\} .
$$

Hence, $u_{i}(x)=u\left(\|x\|, d_{i}, c\right)$ is a solution to (1.1) with exactly $i$ interior nodal surfaces. Thus, Theorem $\mathrm{A}$ is proven.

\section{REFERENCES}

1. A. Ambrosetti and G. Prodi, On the inversion of some differentiable mappings with singularities between Banach spaces, Ann. Mat. Pura Appl. 93 (1972), 231-246.

2. A. Castro and A. Kurepa, Energy analysis of a nonlinear singular differential equation and applications, Rev. Colombiana Mat. 21 (1987), 155-166.

3. __ Infinitely many radially symmetric solutions to a superlinear Dirichlet problem in a ball, Proc. Amer. Math. Soc. 101 (1987), 57-64.

4. A. Castro and R. Shivaji, Multiple solutions for a Dirichlet problem with jumping nonlinearities, Trends in Theory and Practice of Nonlinear Analysis, V. Lakshmikantham (ed.), North-Holland, 1985. 
5. __, Multiple solutions for a Dirichlet problem with jumping nonlinearities II, J. Math. Anal. Appl. 133 (1988), 509-528.

6. A. Castro and A. C. Lazer, On periodic solutions of weakly coupled systems of differential equations, Boll. Un. Mat. Ital. (5) 18-B (1981), 733-742.

7. D. G. Costa and D. G. De Figueiredo, Radial solutions for a Dirichlet problem in a ball, J. Differential Equations 60 (1985), 80-89.

8. J. Kazdan and F. W. Warner, Remarks on some quasilinear elliptic equations, Comm. Pure Appl. Math. 28 (1975), 567-597.

9. A. Lazer and P. J. McKenna, On the number of solutions of nonlinear Dirichlet problem, J. Math. Anal. Appl. 84 (1981), 282-294.

10. __ On a conjecture related to the number of solutions of a nonlinear Dirichlet problem, Proc. Roy. Soc. Edinburgh Sect. A 95 (1983), 275-283.

11. _ Critical point theory and boundary value problems with nonlinearities crossing multiple eigenvalues, Comm. Partial Differential Equations 10 (1985), 107-150.

12. D. Lupo, S. Solimini and P. N. Srikanth, Multiplicity results for an o.d.e. problem with even nonlinearity, Quad. Matematici (II), no. 112, Dicembre 1985.

13. B. Ruf and S. Solimini, On a class of superlinear Sturm-Liouville problems with arbitrarily many solutions, SIAM J. Math. Anal. 17 (1986), 761-771.

Department of Mathematics, University of North Texas, Denton, Texas 76203

Department of Mathematics, Texas Christian University, Fort Worth, Texas 76129 